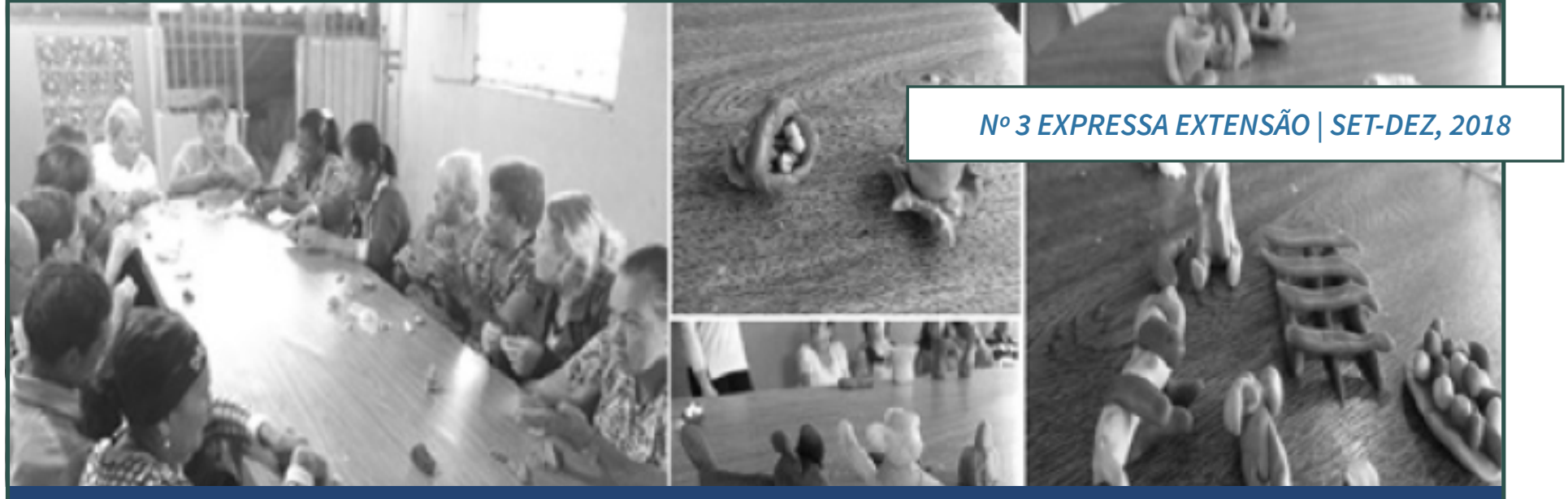

\title{
MEMÓRIAS AUTOBIOGRÁFICAS EVOCADAS POR MÚSICA: OFICINAS PARA IDOSOS COM NÍVEIS DE ESCOLARIDADE E SOCIOECONÔMICO BAIXOS
}

Music-evoked autobiographical memories: Workshops offered to elderly people with low educational and socioeconomic levels.

Katarina Duarte Fernandes ${ }^{1}$ Luisiana Baldini França Passarini ${ }^{2}$ Letícia Tanelli ${ }^{3}$ José Davison da Silva Júnior ${ }^{4}$ Patrícia Maria Vanzella ${ }^{5}$ Maria Teresa Carthery-Goulart ${ }^{6}$

\section{RESUMO}

O projeto ATIVAMENTE-UFABC tem como objetivo estimular a autonomia e melhorar a qualidade de vida dos idosos por meio da oferta de oficinas com diferentes tipos de treinos cognitivos. Os programas desenvolvidos consistem em atividades que buscam otimizar o funcionamento da atenção e memória por meio de ações socioeducativas e atividades teórico-práticas que incluem a aplicação de estratégias para maior funcionalidade e melhor autoestima em relação ao próprio potencial cognitivo. Entre essas ações, temos o AtivAmente-Musical, oficinas que por meio da música promovem o acesso a memórias autobiográficas como mecanismo para treino cognitivo, melhora do humor e resgate da identidade. No presente relato, descrevemos a experiência AtivAmente Musical ofertada a um grupo de idosos de baixo nível educacional e socioeconômico de uma ONG de São Bernardo do Campo/SP. Ao longo de um mês, foram realizadas quatro sessões (uma por semana) de intervenção com atividades musicais que estimulavam evocações de memória autobiográfica. Analisando os resultados dos questioná-

\footnotetext{
${ }^{1}$ Universidade Federal do ABC - Brasil - Discente do Bacharelado em Ciência e Tecnologia e do Bacharelado em Neurociências - kathydfernandes@gmail.com; ${ }^{2}$ Centro de Musicoterapia Benenzon Brasil - Brasil - Bacharel em Musicoterapia com especialização no Modelo Benenzon e pós graduada em Psicopatologia e Saúde Pública e em Reabilitação Cognitiva. Diretora do Centro de Musicoterapia Benezon Brasil - luisiana@centrobenenzon.com.br; ${ }^{3}$ Centro de Musicoterapia Benenzon Brasil - Brasil - Bacharel em Musicoterapia. Pós-Graduação em Gerontologia. Especialização no Modelo Benenzon - letanelli@ hotmail.com; ${ }^{4}$ Instituto Federal de Pernambuco - Brasil - Doutor em Música. Professor dos cursos Técnicos Integrados e do Programa de Pós-graduação em Educação Profissional e Tecnológica - davisonjr@gmail.com; ${ }^{5}$ Universidade Federal do ABC, Núcleo Interdisciplinar de Neurociência Aplicada - Brasil - Professora Doutora do Bacharelado em Neurociências e do PPG em Neurociência e Cognição - Pvanzella@yahoo.com; ${ }^{6}$ Universidade Federal do ABC, Centro de Matemática, Computação e Cognição - Brasil Professora Doutora do Bacharelado em Neurociências e do PPG em Neurociência e Cognição - teresa.carthery@ ufabc.edu.br
} 
rios de sintomas de depressão, ansiedade e testes cognitivos aplicados antes e após a intervenção foi possível verificar uma robusta melhora no estado de ânimo dos participantes. Este projeto é considerado socialmente relevante devido à possibilidade de melhorar a qualidade de vida das pessoas através da intervenção musical e levar conhecimento à comunidade sobre o assunto, além de engajar alunos de graduação e pós-graduação a aprenderem sobre esses assuntos na prática.

Palavras-chave: Memória autobiográfica. Música. Intervenção cognitiva. Humor.

\section{ABSTRACT}

The project ATIVAMENTE-UFABC aims to stimulate autonomy and improve the quality of life of elderly people through different types of memory training. The programs developed consist of activities that seek to optimize the functioning of attention and memory through socio-educational actions and theoretical-practical activities that include the application of strategies for greater functionality and better self-confidence in relation to their cognitive potential. Among these actions, we have the AtivAmente-Musical, a project that use music to help the autobiographical memories access, as a mechanism for cognitive training. The project AtivAmente aims to promote a mood improvement and the recovery of identity. In the present report, we describe the AtivAmente Musical experience offered to elderly people with low schooling and socioeconomic status of an NGO from São Bernardo do Campo / SP. Once a week for one month, we conducted an intervention with musical activities that stimulated evocation of autobiographical memories. Analyzing the results of depression, anxiety, mood and cognitive tests applied before and after the activity we found a robust improvement in the participants' mood. This project is considered socially relevant because it disseminates academic knowledge to the population and improve people's quality of life, as well as engages undergraduate and graduate students (scholarship holders and volunteers) to learn more about these subjects in a practical way.

Keywords: Autobiographical memory. Music. Cognitive intervention. Mood.

\section{INTRODUÇÃO}

O Brasil e o mundo passam por um acelerado processo de envelhecimento populacional. Segundo o relatório “Envelhecimento no Século XXI-Celebração e desafio" publicado pelo Fundo de População das Nações Unidas, estima-se que em 2050 a proporção de pessoas com mais de 60 anos seja de 1 a cada 5, somando aproximadamente 2 bilhões de idosos (UNFPA, 2012). Ainda segundo o mesmo documento, essa elevada taxa de envelhecimento populacional traz à 
tona preocupações e desafios que vão desde políticas públicas visando garantir a essa parcela da população todo o necessário para plena qualidade de vida, como questões relativas a relações sociais e intergeracionais.

Segundo estudos sobre o envelhecimento, existem fortes evidências de que qualidade de vida e independência funcional no dia-a-dia estão intimamente relacionados com as chamadas habilidades cognitivas, tais como atenção, memória, velocidade de processamento, entre outros. Assim, torna-se essencial a promoção de ações para a manutenção da capacidade cognitiva funcional das pessoas idosas, a fim de maximizar suas possibilidades de envelhecimento saudável (BALL et.al., 2002).

As alterações nas capacidades cognitivas associadas ao envelhecimento, representam o ápice de diversas mudanças neurobiológicas que ocorrem ao longo da vida, afetando diferentes áreas do cérebro em níveis diferentes, envolvendo áreas como as corticais pré-frontais e o hipocampo, uma das principais regiões do cérebro relacionadas à memória (KRAMER et.al., 2007).

O estudo do envelhecimento cognitivo humano progrediu expressivamente nos últimos 50 anos. Sabe-se hoje que certos déficits de memória fazem parte do envelhecimento saudável (YASSUDA et al., 2006) e que os déficits de memória em indivíduos idosos saudáveis estão associados com dificuldades na codificação e na evocação das informações. Estudos indicam que os idosos têm uma diminuição em seus recursos de atenção, memória operacional e velocidade de processamento (LEZAK et al., 2004). Entretanto, no envelhecimento saudável existe a possibilidade de algum nível de compensação, mesmo que parcial, dos déficits cognitivos, graças à plasticidade cognitiva, capacidade de adaptação das habilidades funcionais do cérebro (DUNLOSKY; HERTZOG, 1998).

Nesse cenário, as intervenções cognitivas são um meio eficaz de compensar os déficits de memória, principalmente na memória episódica, ou seja, memória para eventos que ocorrem em momentos e locais específicos (CARVALHO, 2010). Há indícios de que o treinamento cognitivo, no geral, pode melhorar a memória, o raciocínio visuoespacial, a atenção e o estado psicológico em idosos, bem como manter mais estável seu funcionamento cognitivo ao longo do tempo, levando a maior independência e autonomia (CHENG et al., 2012). Menciona-se ainda a importância de fatores afetivos para o desempenho cognitivo, considerando-se o impacto de fatores como depressão e ansiedade (YASSUDA et al., 2006). Sabe-se também que variáveis demográficas, como nível de escolaridade, status socioeconômico e gênero afetam o desempenho cognitivo.

A memória como habilidade de reter e usar informações possui três estágios principais, a codificação, o armazenamento ou consolidação e a evocação. A codificação é o processo de formação inicial das memórias a partir de estímulos do ambiente, a consolidação é a passagem dessa codificação de estímulos para 
uma memória de longo prazo, e a evocação é o processo de acessar memórias já consolidadas, que pode se dar espontaneamente ou através do uso de dicas ou pistas ambientais que inicia um processo de associação que facilita a evocação.

Memória autobiográfica é a habilidade de recordar conscientemente uma experiência pessoalmente vivida ou testemunhada, acompanhada de uma sensação de vivenciar novamente o evento original, como uma viagem no tempo (GAUER; GOMES, 2006), envolvendo aspectos sensoriais e emocionais relacionados ao que foi vivido. Encontram-se bem documentadas as diferenças entre adultos jovens e idosos em tarefas envolvendo a memória autobiográfica, com a redução da evocação de detalhes contextuais e perceptuais relevantes em idosos (MCINTYRE; CRAIK, 1987; HASHTROUDI et al., 1990; SPENCER; RAZ, 1995; LEVINE et al., 2002). Em estudos com entrevista estruturada, lembranças autobiográficas de idosos foram classificadas como menos vívidas que as de adultos de meia idade (BORRINI et.al., 1989). Por outro lado, em geral os estudos não mostram declínio significativo em função da idade no domínio da memória semântica, que é um tipo de memória relacionada com o conhecimento geral de mundo que o indivíduo tem, sua "enciclopédia mental" (HAY; JACOBY, 1999; JENNINGS; JACOBY, 1993).

As memórias podem ser evocadas a partir de uma diversidade de estímulos sensoriais, tais como, cheiros, imagens e músicas (olfato, visão e audição) (SILVA JUNIOR, 2016). Para que este estímulo sensorial tenha esta utilidade, ele precisa estar presente na codificação (ANDERSON, 2011), ou estar associado de alguma forma ao contexto da codificação. Memórias autobiográficas evocadas por música podem ser mais intensas que as evocadas por estímulos visuais, como fotos de rostos, uma vez que a música consegue evocar emoções como as sentidas durante o evento lembrado propiciando o efeito de viagem no tempo, o que aumenta a intensidade da memória (BELFI; KARLAN; TRANEL, 2015). Sabe-se também que idosos preferem e têm fortes respostas emocionais à música popular de sua juventude em comparação com música popular de outros períodos (SCHULKIND; HENNIS; RUBIN, 1999).

Nesse contexto, descrevemos a seguir o relato de uma ação do projeto de extensão universitária ATIVAMENTE - UFABC que tem como objetivo estimular a autonomia e melhorar a qualidade de vida dos idosos por meio da oferta de oficinas com diferentes tipos de treinos cognitivos. Os programas desenvolvidos consistem em atividades que buscam otimizar o funcionamento da atenção e memória por meio de ações socioeducativas e atividades teórico-práticas que incluem a aplicação de estratégias para maior funcionalidade e melhor autoestima em relação ao próprio potencial cognitivo. Entre essas açōes, temos o AtivAmente-Musical, oficinas que por meio da música promovem o acesso a memórias autobiográficas como mecanismo para treino cognitivo, melhora do humor e resgate da identidade. 
Como primeira atividade do AtivAmente-Musical, no segundo semestre de 2016 foi feita uma parceria com a Associação de Amparo Social - PROJETO CARIDADE e assim, surgiu a oportunidade de levar as oficinas de intervenção cognitiva para uma população idosa em situação de vulnerabilidade social. Portanto, essa experiência extensionista teve como objetivo adaptar um experimento de estimulação da memória autobiográfica através de uma abordagem musical, já delineado por Silva Junior, para a população idosa de baixa escolaridade e baixa classificação socioeconômica da região de São Bernardo do Campo-SP, visando contribuir com a melhora da qualidade de vida desse público.

\section{METODOLOGIA}

Inicialmente foi feito um levantamento sobre a metodologia utilizada por Silva Junior (2016) em sua tese de doutorado com intervenção musical com foco na memória autobiográfica em idosos, e então foram estudadas quais as modificações e adaptações que deveriam ser feitas para adequar a oficina ao perfil dos participantes e para ser realizada em grupo, dada a estrutura e tempo disponíveis na ONG.

A amostra foi composta por vinte e cinco dos idosos que são atendidos na Associação Projeto Caridade na cidade de São Bernardo do Campo-SP, os quais foram convidados a participar do estudo. Destes, quatro não compareceram em ao menos três dos quatro encontros, e, portanto, não foram incluídos no estudo. O grupo participante contou com vinte e um idosos assíduos, dezenove mulheres e dois homens, com idade média de $67,48( \pm 4,52)$ anos, escolaridade média de dois anos e pertencentes majoritariamente à classe $C$, para mensurar esta informação foi usado o sistema de pontos do questionário do documento "Critério de Classificação Econômica Brasil” de 2016, publicado pela Associação Brasileira de Empresas de Pesquisa (ABEP).

A ação foi dividida nas fases de avaliação cognitiva antes e após a intervenção e na intervenção em si, feita em quatro encontros de aproximadamente duas horas, realizados semanalmente. O cronograma seguido foi o seguinte:

Mutirão de avaliação antes e depois da atividade: Foi feita a aplicação dos questionários e testes previstos, de forma individual pelos membros da equipe, através dos quais realizou-se o levantamento de perfil sociodemográfico. Foram aplicadas escalas geriátricas de sintomas de depressão (GDS) e ansiedade (GAI); uma avaliação cognitiva breve (mínimo exame de estado mental - MEEM), teste de fluência verbal para letra $P$ e animais, escala de atenção e orientação, teste de memória de figuras (NITRINI et.al., 1994; 2004; 2007); os subtestes de dígitos direto e inverso (memória de curto prazo) e de símbolos (velocidade) da escala 
WAIS III e escala de ânimo adaptada conforme demonstrado na figura 1 abaixo. Os mesmos testes foram reaplicados após cinco semanas, ou seja, uma semana após o término da oficina. Os resultados dessas escalas foram analisados por testes pareados para verificar o efeito que a intervenção usada pode ter nessas medidas.

Figura 1 - Escala de ânimo adaptada, aplicada antes e depois da intervenção musical.

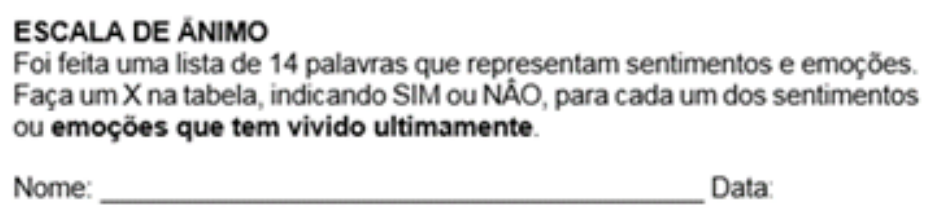

\begin{tabular}{|l|l|l|}
\hline \multicolumn{3}{|c|}{ Antes da atividade } \\
\hline $\begin{array}{l}\text { Emoções } \\
\text { Sentimentos }\end{array}$ & Não & Sim \\
\hline Irritado & & \\
\hline Feliz & & \\
\hline Alegre & & \\
\hline Animado & & \\
\hline Desmotivado & & \\
\hline Angustiado & & \\
\hline Bem & & \\
\hline Deprimido & & \\
\hline Chateado & & \\
\hline Satisfeito & & \\
\hline Nervoso & & \\
\hline Triste & & \\
\hline Contente & & \\
\hline Desanimado & & \\
\hline
\end{tabular}

\begin{tabular}{|l|l|l|}
\hline \multicolumn{3}{|c|}{ Depois da atividade } \\
\hline $\begin{array}{l}\text { Emoções } \\
\text { Sentimentos }\end{array}$ & Não & Sim \\
\hline Irritado & & \\
\hline Feliz & & \\
\hline Alegre & & \\
\hline Animado & & \\
\hline Desmotivado & & \\
\hline Angustiado & & \\
\hline Bem & & \\
\hline Deprimido & & \\
\hline Chateado & & \\
\hline Satisfeito & & \\
\hline Nervoso & & \\
\hline Triste & & \\
\hline Contente & & \\
\hline Desanimado & & \\
\hline
\end{tabular}

Fonte: autores

Encontro 1: Para a definição das três músicas a serem utilizadas como repertório nas oficinas, os idosos ouviram dez músicas pré-selecionadas pela equipe e, ao final da escuta de cada uma delas, indicaram o quanto gostavam ou não da música ouvida através de um questionário com uma escala de cinco rostos do menos feliz ao mais feliz conforme ilustrado na figura 2 abaixo. As três músicas mais votadas e mais pontuadas na escala usada foram utilizadas nas sessões seguintes. Todas as músicas foram extraídas das listas utilizadas na pesquisa original de Silva Junior. 
Figura 2 - Exemplo de questionário usado para selecionar as músicas.

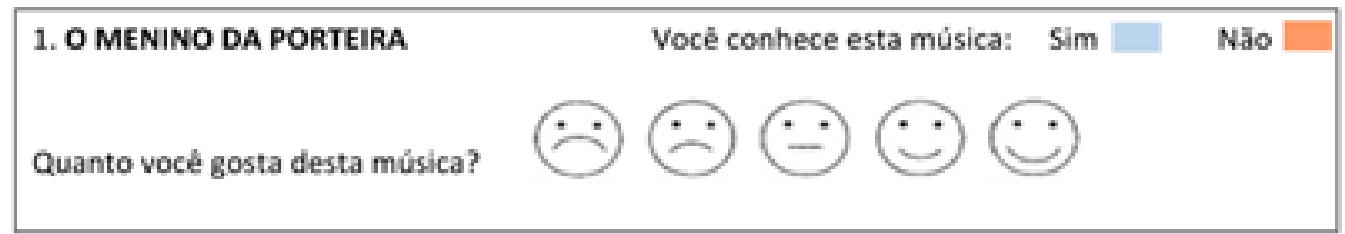

2.MARACANGALHA

Fonte: autores

Encontro 2: Audição da música "Fica comigo esta noite” de Nelson Gonçalves (música mais votada de acordo com o questionário aplicado), em seguida, a equipe fez uma entrevista autobiográfica em grupo, na qual cada um dos idosos falou das lembranças que a música lhe proporcionou.

Encontro 3: Foi realizada uma atividade com massinha de modelar, na qual eles poderiam explorar e modelar livremente com o material, enquanto se tocava repetidamente ao fundo a música "O menino da porteira" de Luisinho e Teddy Vieira (segunda mais votada). Após todos terem finalizado suas produções foi solicitado que cada um falasse sobre o que fez em massinha e em seguida foi feita uma segunda entrevista autobiográfica em grupo de memórias evocadas pela música do dia.

Encontro 4: Foi feita a audição da música "Trem das onze" de Adoniram Barbosa. Na sequência, com um acompanhamento harmônico feito no violão pela equipe, os idosos cantaram e tocaram no pulso da música com pequenos instrumentos de percussão como pandeiros, maracas, entre outros. A atividade focou no 'fazer musical' dos idosos. Novamente a atividade foi seguida de uma entrevista autobiográfica em grupo.

\section{RESULTADOS E DISCUSSÃO}

Ao analisar os questionários e testes feitos antes e depois da intervenção foi possível perceber que houve uma redução estatisticamente significante $(p<0,05)$ para os sintomas depressivos após a intervenção. Já em relação aos sintomas de ansiedade a mudança não teve força estatística. Os resultados encon- 
tram-se no gráfico da Figura 3a. Não houve variações significantes nas pontuações dos demais testes cognitivos realizados, como é possível verificar na Figura $3 \mathrm{~b}$.

Figura 3a - Gráfico boxplot que mostra a distribuição dos resultados das avaliações de sintomas depressivos (GDS) e ansiosos (GAl), antes e depois da intervenção musical, em azul e vermelho respectivamente.

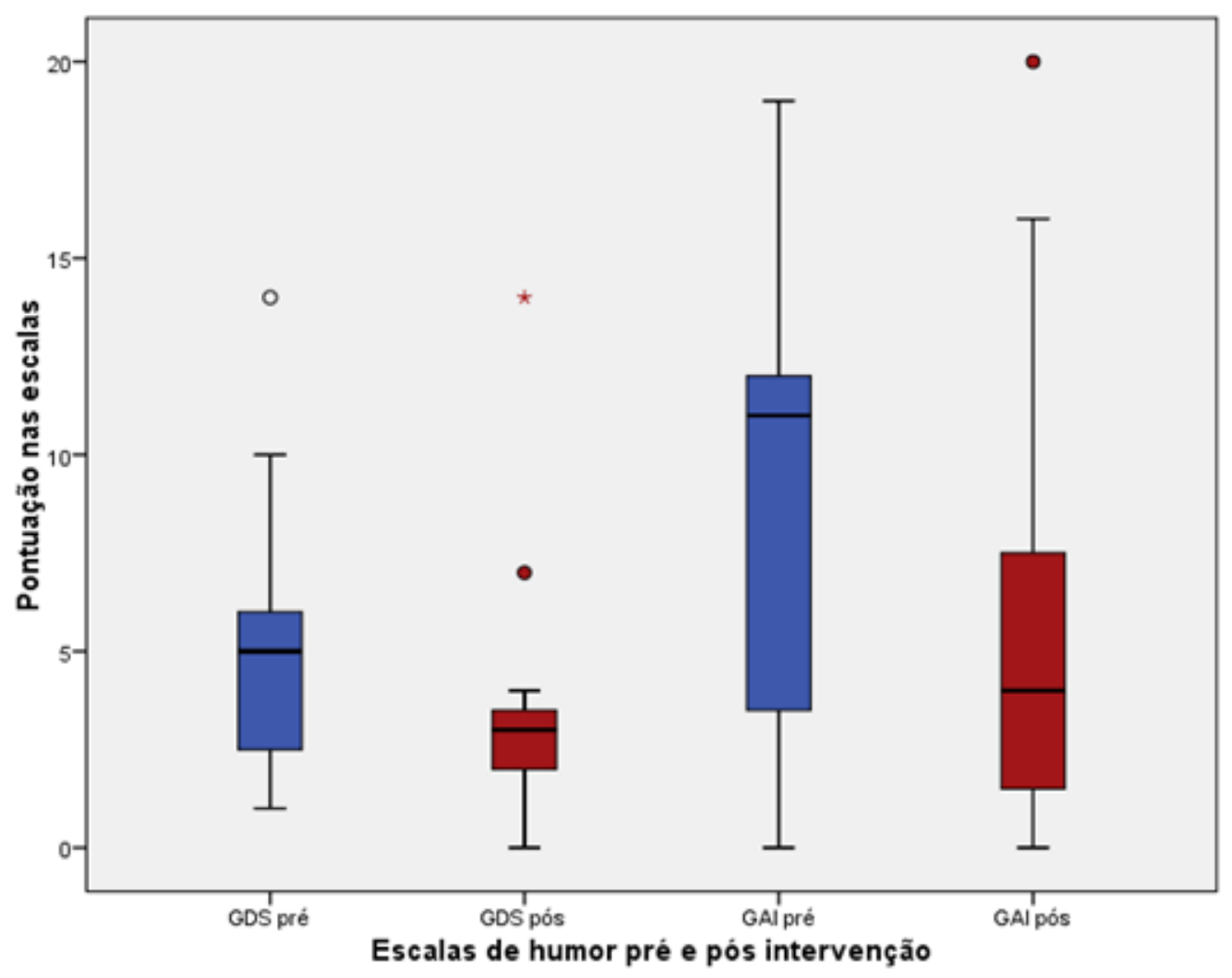

Fonte: autores 
Figura 3b - Gráfico boxplot que mostra a distribuição dos resultados dos testes cognitivos aplicados antes e depois da intervenção musical, em azul e vermelho respectivamente.

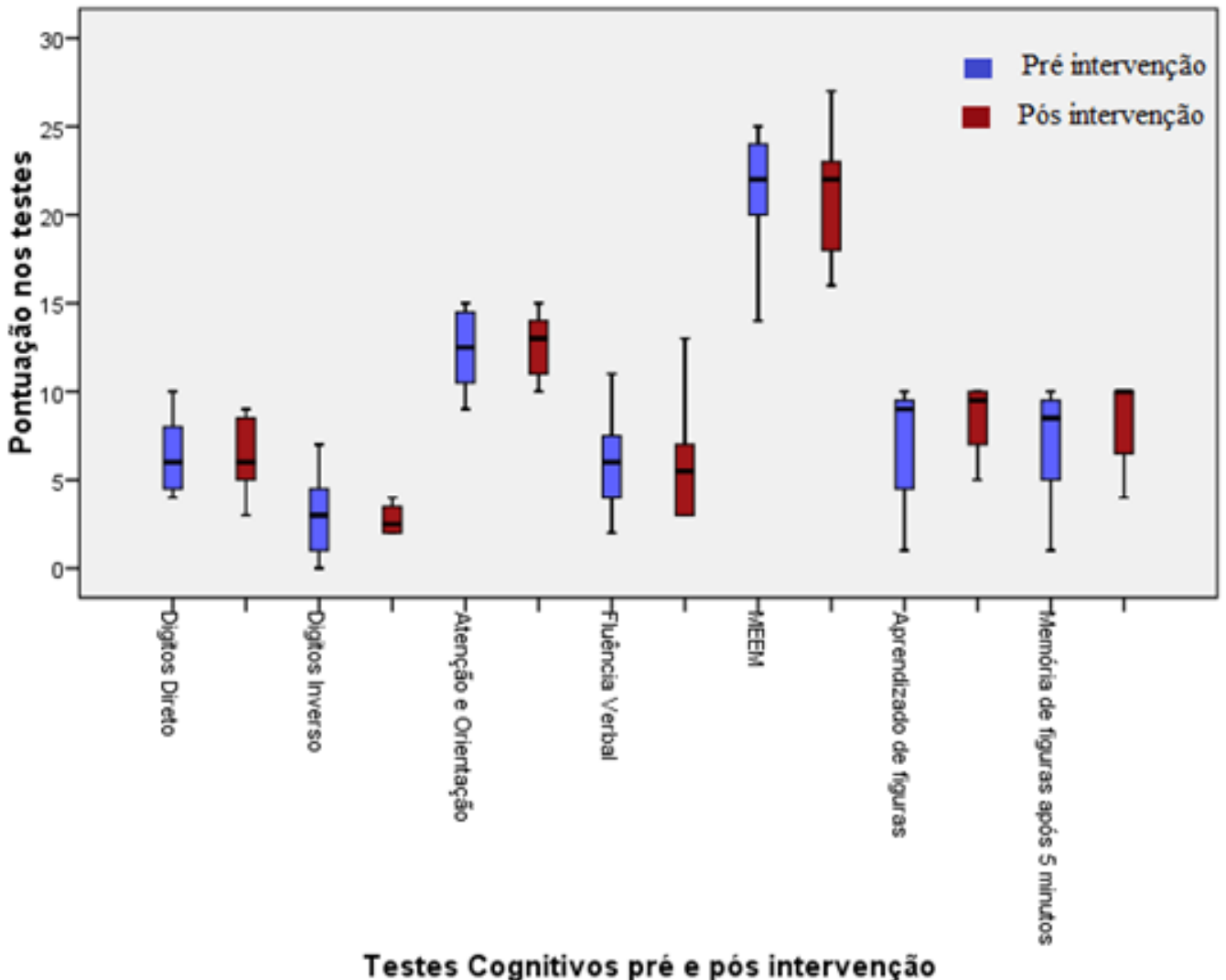

Fonte: autores

Na tabela la podemos verificar que o número de relatos de sentimentos negativos através da escala de ânimo reduziu consideravelmente de antes da intervenção para depois. Para todos os sentimentos essa redução teve significância estatística, com exceção do de tristeza. Já os relatos de sentimentos de valência positiva aumentaram, mas essa mudança só foi significante para o sentimento de felicidade. A frequência do relato desses sentimentos pode ser observada na Tabela 1b. 
Tabela 1a - Quantidade de idosos com sentimentos de valência negativa avaliados pelas escalas de ânimo, antes e depois das atividades musicais realizadas.

\begin{tabular}{|c|c|c|c|}
\hline Sentimento & $\begin{array}{l}\text { Momento da } \\
\text { Avaliação }\end{array}$ & $\begin{array}{l}\text { Sim } \\
\text { (frequência) }\end{array}$ & $\begin{array}{l}\text { Não } \\
\text { (frequência) }\end{array}$ \\
\hline \multirow[t]{2}{*}{ Irritado } & Pré & 9 & 12 \\
\hline & Pós & 2 & 17 \\
\hline \multirow[t]{2}{*}{ Desmotivado } & Pré & 8 & 13 \\
\hline & Pós & 2 & 19 \\
\hline \multirow[t]{2}{*}{ Angustiado } & Pré & 7 & 14 \\
\hline & Pós & 0 & 19 \\
\hline \multirow[t]{2}{*}{ Deprimido } & Pré & 7 & 14 \\
\hline & Pós & 0 & 19 \\
\hline \multirow[t]{2}{*}{ Chateado } & Pré & 9 & 12 \\
\hline & Pós & 1 & 18 \\
\hline \multirow[t]{2}{*}{ Nervoso } & Pré & 12 & 9 \\
\hline & Pós & 0 & 19 \\
\hline \multirow[t]{2}{*}{ Desanimado } & Pré & 5 & 16 \\
\hline & Pós & 0 & 19 \\
\hline \multirow[t]{2}{*}{ Triste } & Pré & 8 & 13 \\
\hline & Pós & 2 & 17 \\
\hline
\end{tabular}

Fonte: autores 
Tabela 1b - Quantidade de idosos com sentimentos de valência positiva avaliados pelas escalas de ânimo, antes e depois das atividades musicais realizadas

\begin{tabular}{|c|c|c|c|}
\hline Sentimento & $\begin{array}{l}\text { Momento da } \\
\text { Avaliação }\end{array}$ & $\begin{array}{l}\text { Sim } \\
\text { (frequência) }\end{array}$ & $\begin{array}{l}\text { Não } \\
\text { (frequência) }\end{array}$ \\
\hline \multirow[t]{2}{*}{ Feliz } & Pré & 17 & 4 \\
\hline & Pós & 19 & 0 \\
\hline \multirow[t]{2}{*}{ Alegre } & Pré & 17 & 4 \\
\hline & Pós & 18 & 1 \\
\hline \multirow[t]{2}{*}{ Animado } & Pré & 18 & 3 \\
\hline & Pós & 19 & 0 \\
\hline \multirow[t]{2}{*}{ Bem } & Pré & 18 & 3 \\
\hline & Pós & 19 & 0 \\
\hline \multirow[t]{2}{*}{ Satisfeito } & Pré & 18 & 3 \\
\hline & Pós & 19 & 0 \\
\hline \multirow[t]{2}{*}{ Contente } & Pré & 18 & 3 \\
\hline & Pós & 19 & 0 \\
\hline
\end{tabular}

Fonte: autores

Como é possível observar, as mudanças de humor obtidas com a intervenção foram muito mais expressivas do que possíveis alterações em outros domínios cognitivos, uma vez que praticamente todas as medidas de humor feitas mostraram uma melhora, mesmo que nem todas tenham diferença estatística. É sabido que tanto a memória autobiográfica quanto experiências musicais têm um forte componente emocional envolvido, e essas mudanças de estado emocional de antes da participação dos idosos na oficina do AtivAmente-Musical pode ser explicada tanto por esse fator, uma vez que as músicas usadas foram as que a princípio lhes traziam mais sentimentos positivos, quanto por se verem em uma posição de protagonismo durante as atividades. 
Muitos dos participantes relataram que a oficina ofereceu novas experiências como brincar com massinha de modelar (Fig. 4) e manusear diferentes instrumentos, coisas que nunca haviam tido a oportunidade de fazer, e através dessas experiências relataram que se sentiram crianças novamente. Outro fator colaborativo foi a formação de um ambiente receptivo para compartilharem histórias de vida evocadas a cada encontro, conforme o passar dos encontros mais idosos foram se voluntariando a compartilhar lembranças com mais detalhes com o grupo.

Figura 4: Atividade com massinha e algumas das produções feitas pelos idosos com massinha de modelar.

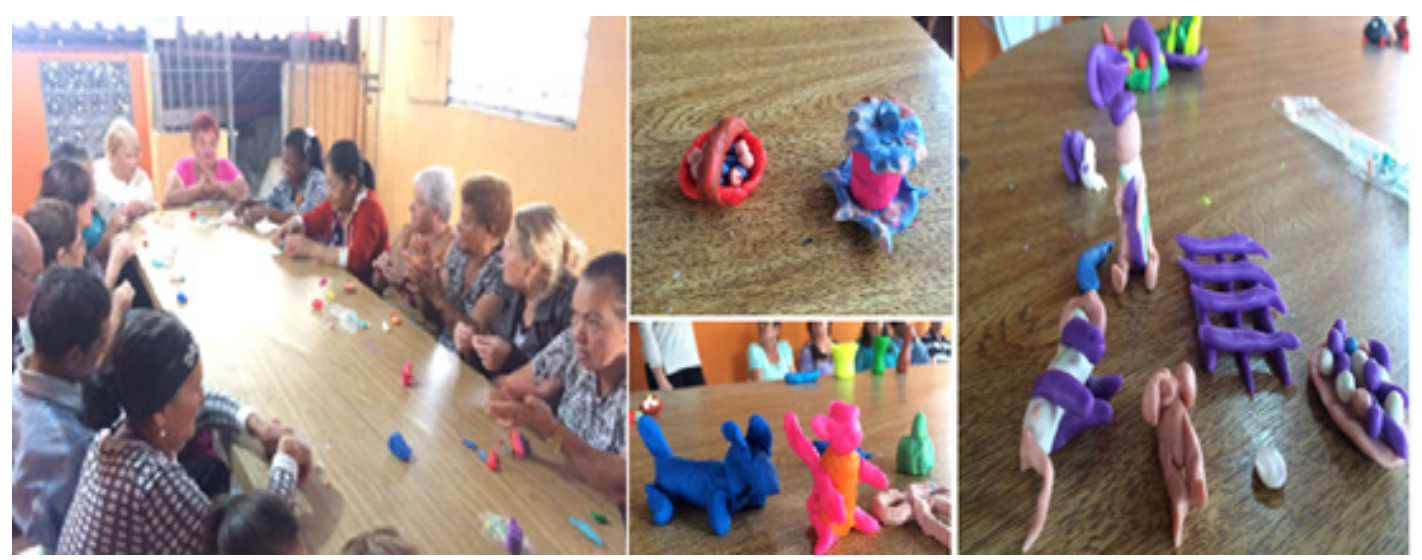

Fonte: autores

\section{CONCLUSÃO}

O projeto teve uma boa adesão entre os idosos da ONG, com pouca taxa de evasão. Dos que permaneceram a participação foi praticamente total em todas as fases do projeto, o que indica que as atividades estimularam e proporcionaram o engajamento por parte do público-alvo.

A partir das avaliações realizadas antes e depois foi possível perceber que houve um impacto positivo na qualidade de vida dessa população em vulnerabilidade socioeconômica, reduzindo a frequência de sentimentos negativos. As oficinas despertaram o interesse dos idosos por assuntos relativos à cognição (memória, atenção, linguagem) no envelhecimento. A partir de discussões em grupo esses temas foram discutidos para contextualizar as atividades realizadas. Essa ação também abriu a possibilidade de novos projetos extramuros do grupo para esse público através da parceria firmada, construindo pontes entre a universidade e a comunidade. 


\section{REFERÊNCIAS}

ASSOCIAÇÃO BRASILEIRA DE EMPRESAS DE PESQUISA (ABEP). Critério de classificação econômica Brasil. 2016. Disponivel em: <http://www.abep.org/criterio-brasil>. Acesso em:18 maio 2018.

BALL, K. et al. Effects of cognitive training interventions with older adults: a randomized controlled trial. Journal of the American Medical Association, v. 288, n. 18, p. 2271-2281, 2002.

BELFI, Amy M.; KARLAN, Brett; TRANEL, Daniel. Music evokes vivid autobiographical memories. Memory, v. 24, n. 7, p. 979-989, 2016.

BORRINI, G. et al. Autobiographical memory:sensitivity to age and education of a standardized enquiry. Psychological Medicine, n. 19, p. 215-224, 1989.

CARVALHO, F. C. R.; NERI, A. L.; YASSUDA, M. S. Treino de memória episódica com ênfase em categorização para idosos sem demência e depressão. Psicologia Reflexão e Crítica, v. 23, n. 2, p. 317-323, 2010.

CHENG, Y. et al. The effects of multi-domain versus single-domain cognitive training in non-demented older people: a randomized controlled trial. BMC Medicine,n. 10, p. 30, 2012.

DUNLOSKY, J.; HERTZOG, C. Aging and deficits in associative memory: what is the role of strategy production? Psychologyand Aging, v. 13, n.4, p. 597-607, 1998.

FERRARI, Elenice A. de Moraes et al. Plasticidade neural: relações com o comportamento e abordagens experimentais. Psicologia: teoria e prática. v. 17, n. 2 , p. 187-194, 2001.

FUNDO DE POPULAÇÃO DAS NAÇÕES UNIDAS (UNFPA);HELPAGE INTERNATIONAL. Envelhecimento no Século XXI: celebração e desafio. 2012. Disponível em:>https://www.unfpa.org/sites/default/files/pub-pdf/Portuguese-Exec-Summary_0.pdf>. Acesso em: 5 mar. 2018.

GAUER, G.; GOMES, W. B. A experiência de recordar em estudos da memória autobiográfica: aspectos fenomenais e cognitivos. Memorandum, v. 11, p. 102-112, 2006. 
HASHTROUDI, S.; JOHNSON, M. K.; CHROSBIAK, L. D. Aging and qualitative characteristics of memories for perceived and imagined complex events. Psychology and Aging, v. 5, p. 119-126, 1990.

HAY, J. F.; JACOBY, L. L. Separating habit and recollection in young and older adults: effects of elaborative processing and distinctiveness. Psychology and Aging, v. 14, p. 122-134, 1999.

JENNINGS, J.; JACOBY, L. Automatic versus intentional uses of memory: Aging, attention, and control. Psychology and Aging, v. 8, p. 283-293, 1993.

KRAMER, J. H. et al. Longitudinal MRI and cognitive change in healthy elderly. Neuropsychology, v. 21, n. 4, p. 412-418, 2007.

LEVINE, B. et al. Aging and autobiographical memory: dissociating episodic from semantic retrieval. Psychology and Aging, v. 17, n. 4, p. 677-689. 2002.

LEZAK, M. D; HOWIESON, D. B.; LORING, D. W. Neuropsychological assessment. Nova Iorque: Oxford University Press, 2004.

MCINTYRE, J. S.; CRAIK, F. I. M. Age differences in memory for item and source information. Canadian Journal of Psychology, v. 41, p. 175-192, 1987.

RAMOS, L. R. Fatores determinantes do envelhecimento saudável em idosos residentes em centro urbano: Projeto Epidoso, São Paulo. Cadernos de Saúde Pública, v. 19, p. 793-797, 2003.

SCHULKIND, Matthew D.; HENNIS, Laura Kate; RUBIN, David C. Music,emotion and autobiographical memory: they're playing your song. Memory \& Cognition, v. 27 n. 6, p.948-955, 1999.

SILVA JUNIOR, José Davison da. Memórias autobiográficas evocadas pela música: um estudo com idosos. Tese (Doutorado em Música). Universidade Federal da Bahia, Salvador, 2016.

SPENCER, W. D., RAZ, N. Differential effects of aging on memory for contentandcontext: a meta-analysis. Psychology and Aging, v. 10, p. 527-539, 1995.

VERHAEGHEN, P. The interplay of growth and decline: theoretical and empirical aspects of plasticity of intellectual and memory performance in normal old age. In: Cognitive rehabilitation in old age. Oxford: Oxford Universit Press, 2000. p. 3-22. 
YASSUDA, M. S. et al. Treino de memória no idoso saudável: benefícios e mecanismos. Psicol.Reflex.Crit.Porto Alegre, v.19, n. 3, p. 470-481, 2006.

Data de recebimento: 05 de março de 2018.

Data de aceite para publicação: 22 de maio de 2018. 